\title{
Redes que tejen conocimientos: hipermediando la enseñanza en la Universidad
}

Networks that weave knowledge: teaching and hypermedia in the

University

Redes que tecem conhecimento: hipermediação o ensino

na Universidade

MARÍA MerCedes MARTín ${ }^{a}$

\section{Resumen}

La identificación con los personajes y la incomodidad que produce el primer capítulo de la tercera temporada de la serie Black Mirror; "Nosedive" (Caída en picada), nos inspira, reta y... ¿obliga? a pensar las redes y las vinculaciones que las mismas habilitan desde una perspectiva más esperanzadora. En este artículo se pretende reflexionar sobre las potencialidades de la inclusión de experiencias con redes sociales en la enseñanza universitaria. Una enseñanza que, inmersa en la cultura digital nos obliga a repensar las propuestas educativas para que se conformen en caminos en los que se tejan relaciones que superen los "puntos" y "likes" como manera exclusiva de comunicarse. Creemos que, estas relaciones mediadas e hipermediadas vinculan personas, conocimientos, grupos, intereses... Black Mirror nos presenta un mundo distópico y pertubador. Proponemos ante estos escenarios experiencias pedagógicas potentes, interesantes y productivas que construyan, al decir de Paulo Freire, "sueños posibles" que nos exijan descubrimiento y utopías en clave histórica. En este sentido se desarrollan algunas de las experiencias sobre las que aquí reflexionaremos.

Palabras clave: Redes. Utopías. Distopías. Experiencias Pedagógicas.

\section{Abstract}

The identification with the characters and the discomfort produced by the first episode of the third season of the Black Mirror series; "Nosedive", inspires us, challenges and ... does it oblige?

\footnotetext{
a Universidad Nacional de La Plata, Ciudad de La Plata, Buenos Aires, Argentina. Mestre em Processos Educativos, e-mail: mmercedesmar@gmail.com
} 
to think about the social networks and the links that they enable from a more hopeful perspective. This article aims to reflect on the potential of the inclusion of experiences with social networks in university education. A teaching that, immersed in the digital culture forces us to rethink the educational proposals so that they conform to paths in which relationships that exceed the "points" and "likes" are woven as the exclusive way to communicate. We believe that these mediated and hypermediated relationships link people, knowledge, groups, interests ... Black Mirror presents a dystopian and disturbing world. We propose to these scenarios powerful, interesting and constructive pedagogical experiences that build, according to Paulo Freire, "possible dreams" that require discovery and utopias in a historical key. In this sense, some of the experiences on which we will reflect here are developed.

Keywords: Social Networks. Utopias. Dystopias. Pedagogical Experiences.

\section{Resumo}

A identificação com os personagens e o desconforto produzido pelo primeiro capítulo da terceira temporada da série Black Mirror; "Nosedive" (Swoop), nos inspira, desafios e ... forças? pensar nas redes e nos links que eles permitem de uma perspectiva mais esperançosa. Este artigo tem como objetivo refletir sobre o potencial da inclusão de experiências com redes sociais no ensino universitário. Um ensino imerso na cultura digital nos obriga a repensar as propostas educacionais para que sejam formadas de maneira que as relações que excedam os "pontos" $e$ os "gostos" sejam tecidas como forma exclusiva de comunicação. Acreditamos que essas relações mediadas e hipermediadas vinculam pessoas, conhecimentos, grupos, interesses ... 0 Black Mirror nos apresenta um mundo distópico e perturbador. Propomos a esses cenários experiências pedagógicas poderosas, interessantes e produtivas que constroem, segundo Paulo Freire, "sonhos possíveis" que exigem descobertas e utopias em termos históricos. Nesse sentido, algumas das experiências sobre as quais refletiremos aqui são desenvolvidas.

Palavras-chave: Redes. Utopias. Distopias. Experiências pedagógicas.

\section{Introducción}

Las tecnologías digitales han llegado a nuestras vidas atravesándolas, modificándolas, facilitando muchas de sus aristas pero complejizando otras. Nos ponen cara a cara ante situaciones inéditas. Nos gustan, nos seducen, nos atemorizan y muchas veces proponen escenarios para los que no tenemos respuestas construidas.

Los objetos culturales de la época problematizan lo contemporáneo y comienzan a reflejar las situaciones referidas... En los mismos se manifiesta una marcada relación entre las ideas a comunicar y las formas en los que se llevan adelante. Las series, se han 
constituido como uno de los objetos culturales contemporáneos más valorados socialmente. Cuentan historias, las recrean, apelan al pasado, al futuro, a la realidad y a la fantasía, al amor, al humor y al terror, nos atrapan, nos entusiasman, también nos decepcionan pero a casi nadie dejan indiferente. Entre otros vínculos interpretan de maneras diversas cómo nuestras vidas se relacionan con las tecnologías:

Erik Brynjolfsson y Andrew McAfee (2014) distinguen un punto de inflexión en el que tecnologías de ciencia ficción empiezan a ser realidad debido a que el progreso tecnológico, que durante mucho tiempo se desarrolló de forma gradual, se volvió veloz. Este fenómeno es atribuido al carácter digital, exponencial y combinatorio de las tecnologías. La innovación digital es innovación re-combinatoria, a la manera una construcción basada en bloques de innovación (MAGGIO et al. 2017, p 4).

En el caso que nos ocupa, es emblemática la mirada de Black Mirror sobre estas relaciones y sus efectos. Una mirada que interpela nuestras prácticas con los usos que las tecnologías digitales nos proponen pues nos presenta un mundo distópico, angustiante y pertubador. "Black Mirror propone un futuro dominado por la distopía. La distopía es la antiutopía. Un pesimismo profundo respecto de lo que vendrá. No ya la visión esperanzada de un mañana mejor (propia de la utopía). No.” (IERARDO, 2018, p. 23).

Quienes nos dedicamos a la educación en general y estudiamos los escenarios de enseñanza mediados con tecnologías digitales nos enfrentamos a estos contextos con una gran "tensión fundante": articular toda la potencia utópica que nos propone la educación en sí misma como una alternativa emancipadora, igualadora, "humanizante" con los escenarios desesperanzados y apocalípticos que las distopías nos presentan.

Todo empieza con un impulso. Un impulso hacia un lado o hacia otro. Desde cierto estado de inmovilidad uno decide iniciar el movimiento. Esto es así en lo social y también en lo educativo. Tenemos que decidir por tanto si ir en pos de la utopía o caer en la distopía. Al fin y al cabo, lo más probable es que sigamos estando hanging in the balance pero será importante reflexionar sobre hacia dónde queremos ir y cómo queremos hacerlo (CARRERA, 2018, p. 38).

\section{Educar en contextos culturales digitales}

Nuestros modos de vivir, de comunicarnos, de participar, de consumir, de relacionarnos, los desafíos ante los que la cotideaneidad nos enfrenta se han transformado 
a partir de la convivencia con las particulares condiciones que han impuesto e-n nuestras existencias las tecnologías digitales.

No se trata de compartir una única monocultura globalizada, sino de una hibridación que no deja de tener en su centro la propia experiencia vivida, pero ahora tensada por un horizonte planetario de sentidos. La mundialización de las comunicaciones, que ha globalizado la circulación de bienes simbólicos, impacta directamente en la subjetividad proponiendo modas, imágenes, formas de ser y consumos de marcas y emblemas que definen el lugar de cada uno en la sociedad. Marcas que se esfuerzan por vender un estilo de vida más que un producto. Y los adolescentes son especialmente sensibles a estos estímulos (BALARDINI, 2002, p. 5).

¿Cómo creer que los modos de enseñar (y de aprender) podrían mantenerse fuera de estas nuevas configuraciones? Y lo mismo podríamos decir de las instituciones que contienen estos procesos tengan la edad que tengan sus sujetos educativos y los preparen para lo que los preparen.

Aquí nos referimos a modos de enseñar que aborden las complejidades de la cultura digital, una cultura hipertextual, interactiva, colectiva, conectada y portable. Estas propuestas deben ser necesariamente desafiantes, inmersivas, donde las tecnologías digitales no sean concebidas como una mera herramienta sino como la cultura que contiene dichas experiencias educativas. Una propuesta educativa es mucho más que una propuesta didáctica, se constituye con aspectos pedagógicos, culturales, políticos, sociológicos, económicos, tecnológicos, etc. Como ya dijimos las tecnologías digitales atraviesan y modifican las sociedades pero no pueden "per se" resolver muchas de las problemáticas actuales: la distribución social del conocimiento, las brechas digitales, las desigualdades de variada índole... pero nuestra hipótesis es que integrarlas críticamente a la educación potencia las posibilidades formativas y se constituye como un factor importante para ser ciudadanos digitales. Aprender con tecnologías ofrece oportunidades cognitivas diferentes a aprender sin ellas. "Enseñar a trabajar con tecnologías como instrumentos de la cultura implica mediatizarlas, al tiempo que configurar particulares relaciones con los entornos físicos y sociales" (LITWIN, 2005, p 23). Ya en estas épocas creemos que es necesario avanzar y profundizar estos procesos proponemos tal como lo hace Scolari (2008) hipermediar la enseñanza, es decir, a proponer procesos de producción, intercambio y consumo de los objetos culturales que se producen en el marco de la cultura digital caracterizada por sujetos, medios y lenguajes interconectados tecnológicamente en una trama compleja. 


\section{“Nosedive" en Black Mirror. ¿Caída en picada?: miradas}

distópicas

Nosedive: caer - desplomarse - descender - caída súbita - vuelo en picada. Son éstos algunos de los significados que encontramos para este término, título del primer capítulo de la tercera temporada de Black Mirror.

Ser prisioneros de nuestros dispositivos. Y de los modos de relacionarse que ellos proponen. Parte de la pesadilla que se propone en "Nosedive"1. "Lo distópico es el concepto clave para situar Black Mirror como referente del pesimismo frente a los sueños idílicos contemporáneos de la vida consumista y cibernética. Y, en particular, su perfil es el de la distopía tecnológica" (IERARDO, 2018, p. 25)

Lacie Pound, protagonista del capítulo, transita su vida en un escenario donde todos, al encontrase deben "calificar" y "ser calificados" a través de sus teléfonos que “apuntan” hacia los otros. Nunca se alejan de sus dispositivos. Además, el puntaje que cada uno obtiene en sus interacciones sociales es público y puede verse a través de unas lentes especiales que se portan en los ojos. Lo que en el "pasado" se realizaba sobre las publicaciones en las redes sociales, ahora se realiza directamente sobre las personas y es parte de su carta de presentación, del capital simbólico que ahora se constituye con el puntaje en estrellas que los otros nos asignan. Estos puntajes constituyen un promedio donde el "5" es el ideal deseado. La máxima cantidad de estrellas que se pueden obtener.

Pero el puntaje no es solamente una apreciación social sino que, a partir del mismo se autorizan o no determinadas acciones, se obtienen ventajas o se inhabilita el acceso a lugares, bienes y / o beneficios. ¿Alguna relación con algunos aspectos o propuestas en nuestra vida actual?

Pensémoslo...

Lacie quiere mejorar su vida y para ello necesita mejorar su puntuación. Cuando una antigua compañera de la escuela la invita a ser su dama de honor y dar un discurso en

\footnotetext{
${ }^{1}$ Primer capítulo de la tercera temporada de Black Mirror protagonizado por la actriz Bryce Dallas Howard, está basada en una historia original de Charlie Brooker siendo adaptado a la televisión por Rashida Jones y Michael Schur. Fue dirigido por Joe Wright y estrenado el 21 de octubre de 2016.
} 
su boda, ella percibe de inmediato la oportunidad: su amiga y sus invitados tienen altos puntajes y es su oportunidad de ser bien calificada por quienes están bien calificados.

Esta "oportunidad" la llevará a emprender un viaje "desastroso" para su puntuación en cuyo final terminará en la cárcel y sin sus lentes para ver las calificaciones de los otros como primera impresión: paradójicamente un viaje a su salvación y a la mirada auténtica sobre las personas.

¿Es sorprendente este enfoque? ¿Es ciencia ficción? Seguramente que así como está planteado, aún sí. Pero varias de sus referencias nos llevan a pensar varias veces en una práctica educativa como lo es la evaluación, que si bien no es el foco único de este artículo no podemos dejar de mencionar. Cuando en las evaluaciones reina lo cuantitativo y no se deja espacio para lo cualitativo, se lo considera menor, se desconfía de sus procedimientos o se combinan pero siempre la primacía la tendrán "las notas" (¿las “estrellas" asignadas por el profesor?) ¿Cuántas veces hemos "calificado" a nuestros estudiantes o hemos mirado y analizado sus calificaciones anteriores suponiendo que eso nos decía más de ellos que sus conversaciones, sus impresiones, sus dudas, sus preguntas, gestos y / o acciones? ¿Estamos construyendo en la escuela y / o la universidad escenarios distópicos? Esos que tanto nos asustan, nos espantan, rechazamos o criticamos cuando nos enfrentamos a las historias que nos los muestran... ¿Son las distopías una amenaza potencial o cuando las profundizamos comenzamos a reconocer algunos de sus componentes en la cotidianeidad?

Preguntas... difíciles pero necesarias. Interrogantes que nos llevan a revisar las prácticas a cuestionar nuestras premisas, a dudar, que ya sabemos... es la acción - fuente del conocimiento...

\section{Redes, likes y conexiones: experiencias educativas}

Como educadores no podemos sucumbir al miedo. La cultura digital nos enfrenta a escenarios desconcertantes y muchas veces inesperados. La identificación con los personajes y la incomodidad que produce el capítulo analizado, nos inspira, reta y... ¿obliga? a pensar las redes y las vinculaciones que las mismas habilitan desde una perspectiva más esperanzadora.

Como hemos dicho, pretendemos aquí reflexionar sobre las potencialidades de la inclusión de experiencias con redes sociales en la enseñanza universitaria. Una enseñanza 
que, inmersa en la cultura digital nos obliga a repensar las propuestas educativas para que se conformen en caminos en los que se tejan relaciones que superen las "estrellas", los puntos y los "likes" como manera exclusiva de verse, valorarse y comunicarse. Creemos que, estas relaciones mediadas e hipermediadas vinculan personas, conocimientos, grupos, intereses, le dan a los jóvenes oportunidades para protagonizar las historias que proponemos en cada clase. Cambian los soportes, entran en foco nuevas herramientas, se potencian las posibilidades de comunicación, de interacción más allá de los espacios y el tiempo pero la cuestión educativa con sus particularidades sigue allí, para ser estudiada, analizada, mejorada.

Las prácticas culturales cotidianas de los jóvenes implican numerosas interacciones mediadas tecnológicamente por recursos digitales. Ello los lleva a configurar una visión del mundo en la que ambos elementos, interacción-digitalización, están interrelacionados. Esta crea matices y niveles de interacción, cuya tipificación son lo que buscamos realizar en este estudio. Mucho se ha escrito sobre las redes sociales digitales y se ha analizado acerca de su papel en movimientos políticos, ciudadanos y sociales (CRUZ ROSALES, et al. 2017, p. 43).

Presentaremos aquí de manera breve algunos usos de las redes en la enseñanza universitaria. En este caso nos referimos a introducir redes sociales en las propuestas de la cátedra de Tecnología Educativa. Esta cátedra, en la que me desempeño como profesora, pertenece al cuarto año de la carrera de Ciencias de la Educación de la Facultad de Humanidades y Ciencias de la Educación de la Universidad Nacional de La Plata (UNLP). La misma se propone como un espacio de formación profesional y de reflexión académica para los y las estudiantes de Ciencias de la Educación entendiendo la importancia de construir conocimientos sobre las transformaciones socio-culturales y educativas actuales atravesadas por el uso casi universal de las tecnologías digitales, interpelar los saberes y las prácticas pedagógicas a partir de la inclusión de tecnologías y acercarse a la interpretación y diálogo con los nuevos lenguajes que surgen en estos contextos. La asignatura es parte de la formación de las y los futuros profesionales en Ciencias de la Educación y propone un recorrido conceptual capaz de abordar las distintas cuestiones que estructuran los contenidos propios de la tecnología educativa de modo crítico, estudiando y analizando influencia de las tecnologías en los procesos de enseñanza, en las instituciones del sistema educativo en todos los niveles y en otros ámbitos en las que las mismas impactan. 
Extiende el aula presencial con un aula virtual en el campus de la facultad, un grupo cerrado en Facebook, diversas herramientas de la web 2.0 y un hashtag ${ }^{2}$ en redes sociales: \#TecnoEduUNLP. Este hashtag tiene dos antecedentes: uno anterior de la misma asignatura: \#TecEdUNLP2014, en el que se puso en marcha una experiencia para "el desarrollo de capacidades críticas para la inclusión de tecnologías digitales en la enseñanza" (Martín, 2014, p.3) y el de la cátedra de Fundamentos de Tecnología Educativa de la Universidad de Buenos Aires (UBA): \#MovimientoTecnoEdu. Este "movimiento" define sus relaciones pedagógico-didácticas con las redes sociales de esta manera:

Si la combinación de aplicaciones en clase suele ser materia de exploración, en nuestras clases es una clave que define cada una de las propuestas a partir de aplicaciones que se usan de forma recurrente (por ejemplo, las asociadas a servicios de redes sociales) y otras para sostener sucesos específicos (por ejemplo, los sistemas de video-conferencia en línea). Ciertos objetos culturales contemporáneos como las películas cinematográficas, las series de televisión y los juegos en línea que capturan la atención de millones de personas se destacan por un rasgo común: la alteración de las formas narrativas lineales. A diario vivimos estas experiencias, definidas por sus alteraciones en torno del tiempo, lo que incluye prospecciones, retrospecciones, simulaciones del tiempo real, suspensiones y paralelismos (MAGGIO, 2015) (MAGGIO, et al. 2017, p. 4-5).

En el caso de \#TecnoEduUNLP, la elección de poner en contacto a las y los estudiantes con herramientas digitales en situaciones de enseñanza no es aleatoria ni caprichosa: responde al interés de formar usuarios críticos, pero no usuarios comunes sino futuros profesionales de la educación que formarán educadores de todos los niveles del sistema educativo. Por ello además del uso, se realizan propuesta de análisis metacognitivo para facilitar el análisis de las mismas y de las circunstancias y propósitos educativos en los que se propone su incorporación. En el caso puntual del uso de la red social Twitter, fue algo resistido entre los estudiantes. Muchos de ellos no tenían usuario en esa red social y otros le daban un uso ocasional relacionado con el ocio.

A partir de estas inclusiones, fueron generándose experiencias diversas, a saber: conectarse con los autores de los textos leídos en clase. En el ejemplo que traemos a

\footnotetext{
${ }^{2}$ El diccionario de la Real Academia Española (RAE) no incluye el término hashtag en su diccionario. El concepto, que suele traducirse como etiqueta, se emplea para aludir a una cadena de caracteres que se inicia con el símbolo \#, conocido como numeral o almohadilla. En redes sociales se utiliza para indicar la temática de una conversación o de un mensaje. Además permite la creación automática de un hipervínculo que brinda acceso a todos los contenidos que incluyan el hashtag en cuestión.
} 
continuación, se propuso la lectura de un texto de Carina Lion y la autora se puso a disposición en Twitter para responder a las preguntas de los estudiantes. He aquí una transcripción del intercambio:

27 mar.

Mercedes Martín@mmercedesmar:Leyendo y discutiendoreportaje a @carinalion \#TecnoEduUNLP

Carina Lion@carinalion: En respuesta a@mmercedesmar: gracias! Estoy para consultas

Mercedes Martín@mmercedesmar: Han realizado excelentes preguntas y entre ellas hay varias dudas sobre gamificacion e inclusión de videojuegos en el aula... Les conté que estás desarrollando proyectos vinculados... Nos ayudás un poco con ese tema? \#TecnoEduUNLP

Carina Lion@carinalion: Sí, por un lado está la introducción de estrategias de gamificación en clase: diseñar misiones, desafíos, trayectos y recorridos que vayan "ganando puntos" y que puedan fortalecer los aprendizajes, la autoestima y la toma de decisiones

Carina Lion@carinalion: En respuesta a@mmercedesmar: cual?

Mercedes Martín@mmercedesmar: "Hay que romper el aula" de la UNL!"

Otra de las situaciones plantadas se relacionó con la participación en propuestas desarrolladas en redes por otras universidades. En este caso, la Universidad Nacional de Entre Ríos (UNER) propuso una dinámica de Twitter-Chat sobre \#AprendizajeConectivo tal como vemos en estos intercambios:

11 abr.

Mercedes Martín@mmercedesmar: \#TecnoEduUNLP Para ver otras experiencias de aulas sin paredes, aumentadas, extendidas...

Pablo Bongiovanni @pbongiovanni: Hoy 20:30 hora Argentina \#ACED_UNER Quién puede participar? Todo aquel interesado en temas de Aprendizaje y Tecnología. Cómo participar? estar atento al hashtag \#ACED_UNER publicaremos las preguntas ejemplo P1), para responder colocar R1) + hashtag

\footnotetext{
${ }^{3}$ Fuente : https://twitter.com/mmercedesmar/status/1110931183116922880 
Pablo Bongiovanni @pbongiovanni: En respuesta a@mmercedesmar: Bienvenidos \#TecnoeduUNLP! si andan por estos temas, linkeamos con \#ACED_UNER

Pablo Bongiovanni @pbongiovanni: Bienvenida Mercedes! cada "colado" es una maravillosa enseñanza para todos. Si abrimos la clase, sin miedo, podemos recibir invitados que nos enriquecen y amplían nuestros horizontes. Bienvenidos también \#TecnoEduUNLP!

Mercedes Martín@mmercedesmar: En respuesta a @pbongiovanni: Mi nombre es Mercedes. Soy profesora de \#TecnoEduUNLP y "colada" en esta propuesta de \#ACED_UNER \#AprendirajeConectivo. ${ }^{4}$

Por último, se utilizan las redes para trabajar colaborativamente la instancia de evaluación. La misma, que consta del análisis de un caso real, se realiza de manera domiciliaria y es acompañada por el equipo docente en las redes por lo que llamamos \#TipsParcial. Los mismos intentan poner en juego y en práctica lo que Mariana Maggio (2012) denomina como "modos de participación en línea de los estudiantes": buscar, encontrarse y ayudarse, compartir, registrar, organizarse y evaluar... (nos). A modo de ejemplo un pequeño intercambio de \#TipsParcial:

20 jun

Mercedes Martín@mmercedesmar: \#TecnoEduUNLP Recuerden que los criterios de evaluación son: Elaboración conceptual con bibliografía propuesta en el programa - Nivel de análisis y profundización - Precisión de las categorías empleadas - Relación de concepto-teorías-autores - Creatividad y originalidad \#TipsParcial

Mercedes Martín@mmercedesmar: "promover, de modo sistemático y sostenido en el tiempo, proyectos de acceso y formación en tecnología educativa para los docentes, si queremos promover la idea de inclusión genuina de la tecnología en las prácticas de la enseñanza” (Maggio, 2012:117) \#TecnoEduUNLP \#TipsParcial

Mercedes Martín @mmercedesmar: "La articulación entre las TIC y la disciplina a través de las actividades propuestas debe dar lugar a una experiencia de aprendizaje cualitativamente diferente, que resultaría poco factible de alcanzar si se abordara sin las TIC" (Manso,2011:74) \#TecnoEduUNLP \#TipsParcial

\footnotetext{
${ }^{4}$ Fuente: https://twitter.com/pbongiovanni/status/1116482570974302208
} 
Nico@Nicolascsed: Hola profe@mmercedesmar pregunta sobre Mas Savia, ¿esta plataforma nos provee de algun medio de comunicacion a modo de mensajeria? Gracias! \#TecnoEduUNLP

Mercedes Martín@mmercedesmar: En respuesta a@Nicolascsed: Hola Nico! Todos los entornos que soportan aulas virtuales tienen herramientas de mensajería: para mensajes privados (une a une, une a muches, une a todes) y otras tipo cartelera donde el profesor escribe para todes y queda de manera pública \#TecnoEduUNLP \#TipsParcial

Nico@Nicolascsed: Ok, gracias! ¿A qué hora es el limite de la entrega mañana?

Mercedes Martín:@mmercedesmar: En respuesta a @Nicolascsed 23:55! TecnoEduUNLP \#TipsParcial

Mercedes Martín @mmercedesmar: Recreo! Cuento infantil sobre cómo evaluar... "V irtudes Choique" de Carlos Durán bttps:/ / tinyurl.com/y5f5hrv5 Mi frase: "Soy la maestra y debo construir el mundo con estos chicos. Pues entonces, ¿con qué levantaré la patria? ¿Con lo mejor o con lo peor? \#TecnoEduUNLP \#TipsParcial

La finalidad de esta estrategia se concentra en brindar oportunidades para experimentar el trabajo en red y sus ventajas para un momento del proceso educativo que suele proponerse frecuentemente de manera individual y casi secreta... Planteamos una experiencia colectiva en donde el proceso tenga mayor protagonismo que el resultado, donde el afuera de la clase se ponga en diálogo con la clase misma y sus actores, un espacio para encontrarse y ayudarse sin ocultarse.

\section{A modo de conclusión: para seguir pensando...}

Black Mirror nos presenta un mundo distópico y pertubador. Como educadores no podemos desconocerlo pero tampoco sucumbir al pesimismo y al escenario apocalíptico. Como señala Begoña Gros...

Las personas precisan una formación que les ayude a vivir en esta sociedad sin muros evitando la fragmentación, dispersión y un exceso de carga emocional y cognitiva. [...] Los sistemas de formación tradicionales basados en metodologías didácticas muy centradas en lo disciplinar precisan transformarse y combinarse con planteamientos que orienten en el diseño de estos nuevos espacios a través de experiencias basadas en la interacción social, la participación activa y los entornos complejos. La pedagogía como ciencia del diseño es un camino que conviene explorar y desarrollar. Fundamentar lo pedagógico en el diseño implica un razonamiento pedagógico más transparente que permite el diálogo, la participación y la

\footnotetext{
${ }^{5}$ Fuente: https://twitter.com/mmercedesmar/status/1141445580411027457
} 
modificación dinámica de los escenarios de aprendizaje. El papel de las tecnologías digitales es fundamental pero deja de tener sentido preguntarse sobre si estas proporcionan un mayor aprendizaje, ya que resulta imposible prescindir de lo tecnológico. Se trata, eso sí, de dar coherencia a los diseños visibilizando el diseño pedagógico frente a los recursos digitales (GROS, 2015).

Las estrategias presentadas en este artículo no son "recetas" sino el resultado de sucesivos acercamientos espiralados a las diversas realidades de los estudiantes con los que compartimos espacios formativos. Hablamos de acercamientos espiralados porque creemos que en el juego de las cercanías y las distancias se van construyendo reflexiones sobre estos nuevos escenarios y sobre los impactos y complejidades antes los que la cultura digital nos enfrenta.

Nos referimos a "espirales ascendentes de conocimiento", de procesos. Las espirales dan cuenta de movimientos que van reflejando avances y retrocesos superando la linealidad e incorporando construcciones colectivas, formas hipervinculadas, movimientos transformadores. La educación, así entendida, es una salida, lo ha sido históricamente y sostenemos fuertemente su vigencia.

Muchas veces las experiencias exceden los relatos por ello creemos que proponer ante estos escenarios experiencias pedagógicas potentes, interesantes y productivas que construyan, al decir de Paulo Freire, "sueños posibles" y nos exijan descubrimiento y utopías en clave histórica:

El sueño posible exige que piense diariamente en mi práctica; me exige descubrimiento, el descubrimiento constante de los límites de mi propia práctica, lo que implica percibir y demarcar la existencia de lo que llamo espacios vacíos a ser llenados. El sueño posible tiene que ver con los límites de estos espacios y esos límites son históricos. [...] El tema del sueño posible tiene que ver exactamente con la educación liberadora y no con la domesticadora. El tema de los sueños posibles, reitero, tiene que ver con la educación liberadora siempre que sea práctica y utópica (FREIRE, 2015, p. 67).

Sueños, procesos, utopías... Mucho por hacer y descubrir. No desconocer los riesgos y las amenazas de las distopías pero tampoco ignorar la fuerza de las utopías que, como dice Eduardo Galeano $^{6}$, sirven para avanzar y seguir caminando...

\footnotetext{
${ }^{6}$ Eduardo Galeano, fue un periodista y escritor uruguayo, considerado uno de los escritores más influyentes de la izquierda latinoamericana. La entrevista en la que pronunció esta frase puede verse en https://www.youtube.com/watch?v=GaRpIBj5xho
} 


\section{Bibliografía}

BALARDINI, S. Jóvenes, tecnología, participación y consumo, 2002. Disponible en <http://biblioteca.clacso.edu.ar/clacso/gt/20101023013657/balardini.pdf>. Acceso en: 18 jun. 2019.

CARRERA, F. Mundos en desaparición, distopías posibles. Una aproximación al relato "There will Come Soft Rains" de Ray Bradbury desde la didáctica de la lengua y la literatura inglesa y desde la didáctica de la contención y de la creatividad." Nudos. Revista Transdisciplinar de Sociología, Teoría y Didáctica de la Literatura, v. 2, n. 1, p. 36-54, 2018. Disponible en: <https://tinyurl.com/yyxzkv3g>. Acceso en: 23 jun. 2019.

CRUZ ROSALES, V. et al. Desafíos de la cultura digital para la educación. México: Sistema de Universidad Virtual. Universidad de Guadalajara, 2017. Disponible en: <http://biblioteca.udgvirtual.udg.mx/jspui/handle/123456789/1836>. Acceso en: 25 jun. 2019.

IERARDO, E. Sociedad Pantalla. Black Mirror y la Tecnodependencia. Buenos Aires: Continente, 2018.

FREIRE, P.; ARAÚJO FREIRE, M. Pedagogía de los sueños posibles: por qué docentes y alumnos necesitan reinventarse en cada momento de la historia. Buenos Aires: Siglo XXI Editores, 2015.

GROS, B. La caída de los muros del conocimiento en la sociedad digital y las pedagogías emergentes. Education in the knowledge society, v. 16, n.1, 2015, p. 58-68.

LITWIN, E. Tecnologías educativas en tiempos de Internet. Buenos Aires: Amorrortu., 2005.

MAGGIO, M. Enriquecer la enseñanza. Los ambientes con alta disposición tecnológica como oportunidad. Buenos Aires: Paidós, 2012.

MAGGIO, M. Clases fuera de serie. Departamento de Extensión Universitaria del Profesorado de la Universidad Tecnológica Nacional. Revista Digital Aprender para Educar con Tecnología, n. 11, may. 2015. Disponible en: <http://e-ducadores.com/aprenderparaeducar/>. Acceso en: 15 jun. 2017.

MAGGiO, M. et al. (coord.). Tecnoedu2016: Enseñanza universitaria en movimiento. 2017. Disponible en: <https://docplayer.es/68593929-Tecnoedu-ensenanza-universitaria-enmovimiento-tecnoeduuba.html>. Acceso en: 17 jun. 2019.

MARTÍN, M. ¡Saquen los teléfonos!': una experiencia para construir apuntes colaborativos con twitter en el aula universitaria en Ateneo de experiencias. In: IV Foro Internacional de Educación Superior en Entornos Virtuales. Perspectivas y desafíos de la bimodalidad / Marcela Ceballos ... [et al.] Bernal: Universidad Virtual de Quilmes, 2015.

SCOLARI, C. Hipermediaciones: elementos para una teoría de la comunicación digital interactiva. Barcelona: Editorial Gedisa, 2008.

RECEBIDO: $22 / 08 / 2019$

APROVADO: 01/09/2019
RECEIVED: 08/22/2019

APPROVED: 09/01/2019
RECIBIDO: 22/08/2019

APROBADO: 01/09/2019 